\title{
Monte Carlo study of the finite-size effects on the magnetization of maghemite small particles
}

\author{
Òscar Iglesias, ${ }^{a}$ Amílcar Labarta, and Fèlix Ritort \\ Department de Física Fonamental, Universitat de Barcelona, Diagonal 647, 08028 Barcelona, Spain
}

\begin{abstract}
Monte Carlo simulations of a model for $\gamma-\mathrm{Fe}_{2} \mathrm{O}_{3}$ (maghemite) single particle of spherical shape are presented aiming at the elucidation of the specific role played by the finite size and the surface on the anomalous magnetic behavior observed in small particle systems at low temperature. The influence of the finite-size effects on the equilibrium properties of extensive magnitudes, field coolings, and hysteresis loops is studied and compared to the results for periodic boundaries. It is shown that for the smallest sizes the thermal demagnetization of the surface completely dominates the magnetization while the behavior of the core is similar to that of the periodic boundary case, independently of $D$. The change in shape of the hysteresis loops with $D$ demonstrates that the reversal mode is strongly influenced by the presence of broken links and disorder at the surface.

(C) 2001 American Institute of Physics. [DOI: 10.1063/1.1355354]
\end{abstract}

Maghemite $\left(\gamma-\mathrm{Fe}_{2} \mathrm{O}_{3}\right)$ and other magnetic oxides in the form of nanometric particles display anomalous magnetic properties at low temperatures. Experiments have shown that the hysteresis loops display high closure fields and do not saturate $^{1-3}$ even at fields of the order of $50 \mathrm{~T}$. Low magnetization as compared to bulk, shifted loops after field cooling and irreversibilities between the field cooling and zero field cooling processes even at high fields are also observed., ${ }^{2,3}$ Moreover, the existence of aging phenomena ${ }^{4}$ in the time dependence of the magnetization, indicates that there must be some kind of freezing leading to a complex hierarchy of energy levels. Whether these phenomena can be ascribed to intrinsic properties of the particle itself [spin-glass (SG) state of the surface which creates an exchange field on the core of the particle], or they are due to a collective behavior induced by interparticle interactions, ${ }^{5-7}$ has been the object of controversy in recent years and up to the moment there is no model giving a clear-cut explanation of this phenomenology, although simulation results for general small particle systems $^{8}$ and, in particular, for maghemite ${ }^{9}$ have been recently published. In order to contribute to the elucidation of this controversy we present the results of a Monte Carlo (MC) simulation of a single spherical particle which aims at clarifying what is the specific role of the finite size and surface on the magnetic properties of the particle, disregarding the interparticle interaction effects.

Maghemite is a ferrimagnetic spinel in which the magnetic $\mathrm{Fe}^{3+}$ ions with spin 5/2 are disposed in two sublattices with different oxygen coordination [eight tetrahedric $(T)$ and 16 octahedric $(O)$ sites per unit cell]. In our model, the $\mathrm{Fe}^{3+}$ magnetic ions are represented by Ising spins $S_{i}^{\alpha}= \pm 1$ which allows us to reproduce a case with strong uniaxial anisotropy while keeping computational efforts within reasonable limits. The spins interact via antiferromagnetic exchange interactions with the nearest neighbors on both sublattices and with an external magnetic field $H$. In the simulation, we have used the reduced field $h=\mu H / k_{B}$ in temperature units, where $\mu$ is

\footnotetext{
${ }^{a)}$ Electronic mail: oscar@ffn.ub.es
}

the magnetic moment of the $\mathrm{Fe}^{3+}$ ions. The values of the nearest neighbor exchange constants for maghemite are $^{9}$ $J_{T T}=-21 \mathrm{~K}, J_{O O}=-8.6 \mathrm{~K}, J_{T O}=-28.1 \mathrm{~K}$. We have used periodic boundary $(\mathrm{PB})$ conditions to simulate the bulk properties and free boundaries (FB) for a spherically shaped particle with $D$ unit cells in diameter when studying finite size effects. In the latter case, two different regions are distinguished: the surface formed by the outermost unit cells and an internal core. The size of the studied particles ranges from $D=3-14$ corresponding to real particle diameters from 2.49 to $11.62 \mathrm{~nm}$.

We start by studying the effect of FB conditions and finite-size effects on the equilibrium properties in zero magnetic field. The simulations have been performed using the standard Metropolis algorithm. Starting from a very high temperature $(T=200 \mathrm{~K})$ and an initially disordered state with spins randomly oriented, the system was cooled down at a constant temperature step $\delta T=-2 \mathrm{~K}$. After discarding the first $1000 \mathrm{MC}$ steps, the averages of the thermodynamic quantities were computed at each temperature during a number of MC steps ranging from 10000 to 50000 depending on the system size.

In Fig. 1, we compare the thermal dependence of the magnetization for spherical particles of different diameters $D$ with the corresponding results for a system of size $N=14$ and PB (uppermost curve). A second order transition from paramagnetic to ferrimagnetic order signaled by a sharp peak at $T_{c}(D)$ in the susceptibility (see the inset in Fig. 1) is clearly observed. Finite-size effects on both the magnetization and the susceptibility are very important even for $D$ 's as large as 14 in the FB case, with $T_{c}(D)$ increasing as $D$ increases and tending to the value for PB (which varies from 122 to $126 \mathrm{~K}$ when increasing $N$ from 3 to 14). The main feature in Fig. 1 is the reduction of the magnetization $M$ with respect to the PB case (dashed line) due to the lower coordination of the spins at the surface, which hinders perfect ferrimagnetic order at finite temperatures. It is worth noting that for all the studied diameters there is a temperature range, in which the demagnetization process of $M$ is linear, this 


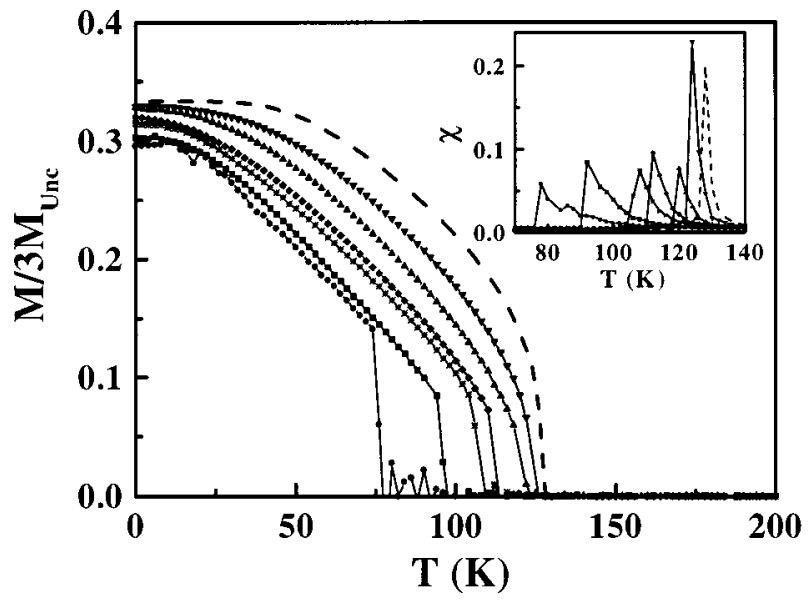

FIG. 1. Thermal dependence of the magnetization $M$. The results for particle diameters $D=3,4,5,6,8,14$ (from the lowermost curve in circles) and PB conditions $N=14$ (uppermost curve) are shown. Inset: Thermal dependence of the susceptibility for the same cases (the PB case is drawn in dashed lines). $M_{\mathrm{Unc}}$ is the ratio of the difference of $O$ and $T$ spins to the total number of spins.

range being wider as the particle size decreases. In this linear regime the particle demagnetization becomes dominated by the surface effects, being the core and surface behaviors strongly correlated. Linear demagnetization is indicative of the effective three-dimensional and two-dimensional reduction of the surface spins and has previously been observed in thin film systems. ${ }^{10}$

Deeper insight into the magnetic ordering of this system can be gained by studying the thermal dependence of the equilibrium magnetization in a magnetic field. Several such curves are presented in Fig. 2. They have been obtained by the same cooling procedure as described previously in the presence of different fields $h_{\mathrm{FC}}$. In this figure, the surface

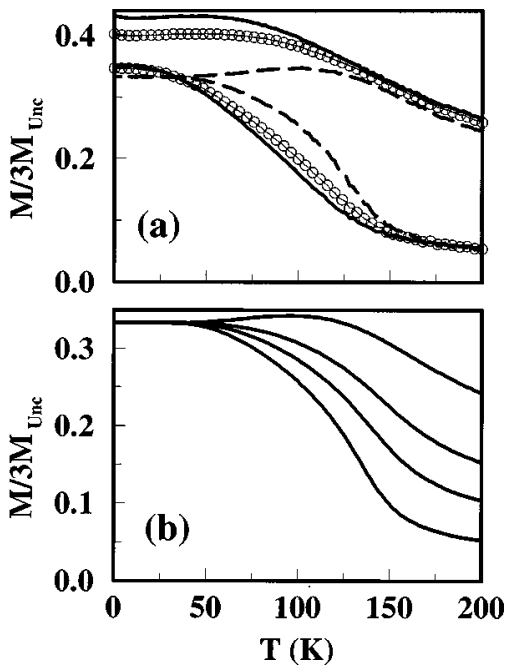

FIG. 2. Thermal dependence of $M$ after cooling under $h_{\mathrm{FC}}$. (a) Corresponds to a spherical particle with $D=6$. The results for two cooling fields $h_{\mathrm{FC}}=20 \mathrm{~K}$ (lower curve) and $h_{\mathrm{FC}}=100 \mathrm{~K}$ (upper curve) are shown. The contributions of the surface (thick lines) and the core (dashed lines) to the total magnetization (circles) have been plotted separately. (b) Shows $M$ for an $N=8$ system and $\mathrm{PB}$ after cooling in $h_{\mathrm{FC}}=20,40,80,100 \mathrm{~K}$ (from lower to uppermost curves). (See Fig. 1 for the definition of $M_{\mathrm{Unc}}$.)
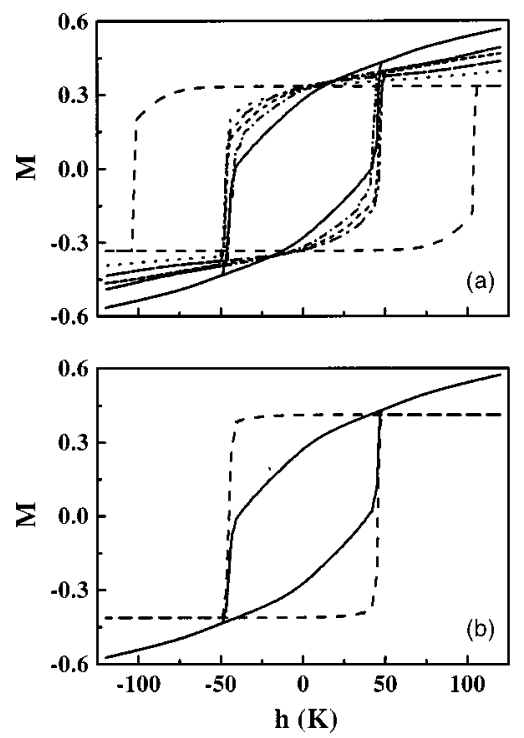

FIG. 3. (a) Hysteresis loops for particles of diameters $D=3,4,6,8,10$ (from inner to outermost curves) and PB (long-dashed lines) at $T=20 \mathrm{~K}$. (b) Surface (continuous line) and core (dashed line) contributions for a particle of diameter $D=3$ at $T=20 \mathrm{~K}$.

(continuous lines) and the core (dashed lines) contributions to the total magnetization have been distinguished. For PB all the curves tend to the ferrimagnetic order value (i.e., $M$ $=1 / 3$ ). A maximum appears at high enough cooling fields $h_{\mathrm{FC}}=100 \mathrm{~K}$ which is due to the competition between the ferromagnetic alignment induced by the field and the spontaneous ferrimagnetic order (as the temperature is reduced the strength of the field is not enough as to reverse the spins into the field direction). However, for particles of finite size the curves at different fields do not converge to the ferrimagnetic value at low $T$, reaching higher values of the magnetization at $T=0$ the higher the cooling field [see lines with circles in Fig. 2(a)]. The total magnetization for small particles is completely dominated by the surface contribution and this is the reason why the ferrimagnetic order is less perfect at these small sizes and the magnetic field can easily magnetize the system. However, the behavior of the core is still very similar to that of the case with $\mathrm{PB}$, although its contribution to $M$ is small. At low fields, the surface is in a more disordered state than the core: its magnetization lies below $M$ at temperatures for which the thermal energy dominates the Zeeman energy of the field. In contrast, a high field is able to magnetize the surface easier than the core due to the fact that the broken links at the surface worsen the ferrimagnetic order while the core spins align towards the field direction in a more coherent way.

In Fig. 3(a), we show the hysteresis loops for several particle diameters at $20 \mathrm{~K}$. The loops have been computed by starting from a demagnetized state at $h=0$ (the first magnetization curve is not shown in this figure). The results have been averaged over several $(\approx 10)$ independent runs starting with different random number seeds. First of all, let us note that the saturation field and high-field susceptibility increase as the particle size is reduced, since these quantities are mainly associated with the progressive alignment of the surface spins towards the field direction. As a consequence, the 
cycles of the smallest particles are similar to those observed in disordered systems, increasing their squareness (uniform rotation of $M$ ) with the size. In fact, by plotting separately the contributions of the core and surface to the total magnetization [see Fig. 3(b)], we see that the loop of the core is almost perfectly squared independently of the particle size, indicating a coherent reversal of its magnetization, while the loop of the surface reveals a progressive reversal of $M$, which is a typical feature of a disordered or frustrated system. Nonetheless, the coercive field of the core is slightly higher but very similar to the one of the surface indicating that the reversal of the surface spins triggers the reversal of the core. We have shown that the existence of lower coordination at the surface of the particle hinders perfect ferrimagnetic order, increasing the magnetic disorder at the surface layer as the size of the particle decreases. However, neither the magnetic frustration associated with the competition between intra- and inter-sublattice antiferromagnetic interactions, finite-size or surface effects are not enough to produce a SG layer contrary to the experimental observation of some authors. ${ }^{1,3}$ This indicates that other ingredients (i.e., enhanced anisotropy at the surface) should be included in the model to account for the SG layer. Our model shows that magnetic disorder at the surface simply facilitates the thermal demagnetization of the particle and also increases the magnetization at moderate fields, since surface disorder diminishes ferrimagnetic correlations within the particle.
The authors we acknowledge CESCA and CEPBA under coordination of $\mathrm{C}^{4}$ for the computer facilities. This work has been supported by SEEUID through Project No. MAT20000858 and CIRIT under Project No. 2000SGR00025.

${ }^{1}$ R. H. Kodama, S. A. Makhlouf, and A. E. Berkowitz, Phys. Rev. Lett. 79, 1393 (1997)

${ }^{2}$ M. García del Muro, X. Batlle, and A. Labarta, Phys. Rev. B 59, 13584 (1999).

${ }^{3}$ B. Martínez, X. Obradors, L1. Balcells, A. Rouanet, and C. Monty, Phys. Rev. Lett. 80, 181 (1998).

${ }^{4}$ T. Jonsson, J. Mattsson, C. Djurberg, F. A. Khan, P. Nordblad, and P. Svedlindh, Phys. Rev. B 75, 4138 (1995); T. Jonsson, P. Nordblad, and P. Svedlindh, ibid. 57, 497 (1998).

${ }^{5}$ X. Batlle, M. García del Muro, and A. Labarta, Phys. Rev. B 55, 6440 (1997).

${ }^{6}$ J. L. Dormann, R. Cherkaoui, L. Spinu, M. Nogués, F. Lucari, F. D'Orazio, D. Fiorani, A. García, E. Tronc, and J. P. Jolivet, J. Magn. Magn. Mater. 187, L139 (1998).

${ }^{7}$ S. Morup and E. Tronc, Phys. Rev. Lett. 72, 3278 (1994).

${ }^{8}$ K. N. Trohidou and J. A. Blackman, Phys. Rev. B 41, 9345 (1990); D. A. Dimitrov and G. M. Wysin, ibid. 50, 3077 (1994); 51, 11947 (1995).

${ }^{9}$ H. Kachkachi, A. Ezzir, M. Nogués, and E. Tronc, Eur. Phys. J. B 14, 681 (2000); H. Kachkachi, M. Nogués, E. Tronc, and D. A. Garanin, J. Magn. Magn. Mater. 221, 158 (2000); R. H. Kodama and A. E. Berkowitz, Phys. Rev. B 59, 6321 (1999).

${ }^{10}$ B. Martínez and R. E. Camley, J. Phys.: Condens. Matter 4, 5001 (1992); G. Xiao and C. L. Chien, J. Appl. Phys. 61, 4313 (1987); A. Corciovei, Phys. Rev. 130, 2223 (1963). 